\title{
Clinical Pearls in Palliative Medicine 2012
}

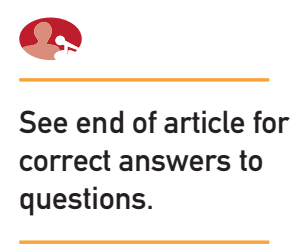

See editorial comment, page 1040

From the Division of General Internal Medicine, Mayo Clinic, Rochester, MN.
A Keith M. Swetz, MD, MA; Elise C. Carey, MD; and John B. Bundrick, MD $t$ the 2001 Annual Conference of the American College of Physicians, a new teaching format to aid physician learning, Clinical Pearls, was introduced. Clinical Pearls is designed with the 3 qualities of physicianlearners in mind. First, we physicians enjoy learning from cases. Second, we like concise, practical points that we can use in our practice. Finally, we take pleasure in problem solving.

In the Clinical Pearls format, speakers present a number of short cases in their specialty to a general internal medicine audience. Each case is followed by a multiple-choice question answered live by attendees using an audience response system. The answer distribution is shown to attendees. The correct answer is then displayed and the speaker discusses teaching points, clarifying why one answer is most appropriate. Each case presentation ends with a Clinical Pearl, defined as a practical teaching point that is supported by the literature but generally not well known to most internists.

Clinical Pearls is currently one of the most popular sessions at the American College of Physicians meeting. As a service to its readers, Mayo Clinic Proceedings has invited a selected number of these Clinical Pearl presentations to be published in our Concise Reviews for Clinicians section. "Clinical Pearls in Palliative Medicine 2012" is one of them.

\section{CASE 1}

A 79-year-old woman with moderate Alzheimer dementia suffered a ground-level fall at her skilled-care facility. She has a history of obesity, essential hypertension (well controlled with oral metoprolol), and chronic renal insufficiency (baseline creatinine level, $2.1 \mathrm{mg} / \mathrm{dL}$ ). On presentation to the emergency department, she is found to have a shortened and laterally rotated right lower extremity. Vital signs include the following: pulse rate, 120/min (regular); blood pressure, 178/98 mm Hg; and respiratory rate, 18 breaths/ min. Hip radiography reveals a displaced femoral neck fracture. The patient is moaning and yelling out with the slightest movement but is not able to rate pain using a visual analog scale or numerical rating scale.

\section{Question}

\section{Which one of the following is the most} appropriate next step in her management?

a. Morphine, $4 \mathrm{mg}$ intravenously once

b. Hydromorphone, $0.4 \mathrm{mg}$ intravenously once

c. Oxycodone, $5 \mathrm{mg}$ orally once

d. Metoprolol, $5 \mathrm{mg}$ intravenously; may repeat every 5 minutes for 3 doses

e. Fentanyl, 12- $\mu \mathrm{g} / \mathrm{h}$ transdermal patch applied immediately

\section{Discussion}

Based on the time required to achieve maximal pain relief and the presence of concurrent renal insufficiency in this patient, the best option is intravenous hydromorphone. This elderly patient presents with a hip fracture. Although it is important to elucidate the patient's goals of care regarding operative management, currently she is in severe pain, as indicated by her agitation, her sensitivity to any movement, her vocalizations, and sympathetic activation due to pain (tachycardia and hypertension). The most appropriate treatment for her severe, acute pain is intravenous opiates titrated to effect. Although intravenous morphine and intravenous hydromorphone at the listed doses are both reasonable starting doses in an opiate-naive patient, this patient has a history of stage 4 chronic kidney disease with an estimated creatinine clearance of $28 \mathrm{~mL} / \mathrm{min}$ per $1.73 \mathrm{~m}^{2}$, making morphine a less desirable choice. ${ }^{1}$ Morphine has active metabolites that accumulate in the setting of renal failure, increasing the risk of opioidinduced neurotoxicity. Therefore, intravenous hydromorphone is the most appropriate choice in this patient.

Oral analgesics are not indicated for this type of severe, acute pain. The time to peak effect of oral opiates, such as oxycodone, is up to 60 minutes, as compared to 6 to 15 minutes for intravenous opiates. The effect of transdermal fentanyl is even more delayed, and it is best used in the setting of chronic, stable pain. Transdermal fentanyl is not indicated for opioid-naive patients. Intravenous fentanyl could be appropriate in this setting, but physicians should be mindful of the short half-life of intravenous fentanyl and utilize an appropriate redosing interval. Metoprolol is a poor choice in that it does not address the etiology of the patient's hypertension and tachycardia, which is most likely pain. 


\section{Clinical Pearl}

Opioids (selected, dosed, and administered appropriately) are indicated for management of moderate to severe pain, even in elderly patients with a history of cognitive impairment and renal insufficiency.

\section{CASE 2}

A 58-year-old woman with idiopathic pulmonary fibrosis presents to your outpatient primary care clinic. She has experienced progressive dyspnea on exertion and reports shortness of breath with minimal activity. Her condition has been relatively stable with use of $4 \mathrm{~L} / \mathrm{min}$ of supplemental oxygen via nasal cannula prior to this visit. The patient does not appear anxious, but she is frustrated that minimal activity triggers dyspnea. Pulmonary examination reveals "Velcro" crackles in all lung fields bilaterally. The following vital signs are recorded in your office: pulse rate, 86/min (regular); blood pressure, 125/77 $\mathrm{mm} \mathrm{Hg}$; and respiratory rate, 16 breaths/min at rest with pulse oximetry at $94 \%$ while the patient received $4 \mathrm{~L} / \mathrm{min}$ of supplemental oxygen, which is unchanged from previous evaluations. After walking from the examination table to a chair in the office, her pulse rate is $125 / \mathrm{min}$, respiratory rate is 24 breaths/min, and pulse oximetry is $91 \%$, and she feels moderately dyspneic.

\section{Question}

\section{Which one of the following is the most appropriate treatment for the patient's shortness of breath?}
a. Haloperidol, I mg orally once
b. Oxycodone, $5 \mathrm{mg}$ orally once
c. Morphine, $2.5 \mathrm{mg}$ preservative-free solution mixed with $2.5 \mathrm{~mL}$ of $0.9 \%$ sodium chloride and inhaled via nebulizer
d. Lorazepam, I mg orally once
e. Change supplemental oxygen delivery system to deliver a higher concentration of oxygen

\section{Discussion}

Multiple studies, including controlled trials and systematic reviews, have demonstrated that systemically delivered opioids are beneficial in the management of dyspnea, ${ }^{2}$ including dyspnea associated with chronic obstructive pulmonary disease and interstitial lung disease as well as with malignant neoplasms. Although major comparison studies among opioids are lacking, given their common effect on the $\mu$-opioid receptor, it is believed that all types of systemic opioids are effective in the treatment of dyspnea, and opioid selection should be based on adverse effects and metabolic considerations for the individual patient.
None of the other medications listed have been convincingly shown to decrease breathlessness. Lorazepam can address anxiety related to dyspnea and can be an effective adjunctive therapy, but it has no direct effect on the sensation of air hunger. Haloperidol may help with agitation but also has no direct mechanism for addressing shortness of breath. Nebulized morphine was not definitively shown to have benefit vs subcutaneous morphine in a case series. ${ }^{3}$ Another case series that focused on nebulized fentanyl demonstrated some potential benefit in a small cohort. ${ }^{4}$ Treating dyspnea with nebulized opiates has great appeal given the ease of administration and the abundance of opioid receptors in the lung, and some patients and physicians believe that they are helpful on the basis of anecdotal information and data from small case series. However, larger randomized, placebo-controlled clinical trials with nebulized opioids are unavailable to support their routine or first-line use for the treatment of dyspnea.

Oxygen therapy has been shown to have beneficial effects on the outcomes of patients with chronic obstructive pulmonary disease and other life-limiting illnesses who are hypoxic, including improved survival, pulmonary hemodynamics, exercise capacity, and neuropsychological function and decreased sensation of dyspnea. However, in nonhypoxic patients, multiple studies have demonstrated that oxygen does not relieve dyspnea. A recent review of the management of dyspnea commented on the equivalence of nasal cannula delivery of oxygen vs nasal cannula delivery of room air in dyspneic patients with life-limiting illness who were not hypoxic. ${ }^{5}$

Compassionate use of oxygen is common in the treatment of dyspnea, even for patients who do not meet criteria for oxygen therapy. Additionally, oxygen is a potent symbol of medical care. However, oxygen therapy is not without burdens, including cost, supply logistics, and flammability. This data may help clinicians guide their patients as they consider the potential benefits and burdens of oxygen therapy for themselves. Some nonhypoxic patients believe that oxygen therapy is helpful and gives them an added feeling of security.

\section{Clinical Pearl}

Systemic opioids (selected and dosed appropriately) are the mainstay of therapy in the treatment of dyspnea refractory to medical management and can be used safely without inducing respiratory compromise. 


\section{CASE 3}

A 70-year-old retired policeman with a 100-packyear history of cigarette use has been diagnosed with stage IV non-small cell lung cancer. His performance status allows him to walk daily, tend to his garden, and perform all activities of daily living. On initiation of chemotherapy with carboplatin and pemetrexed, he experienced some fatigue, anorexia, and chest wall pain at the site of his largest tumor. However, radiographic studies show that the tumors are stable or slightly improved. He, his wife, and his oldest daughter present to you in the primary care clinic after visiting with his oncologist. His granddaughter is getting married in 3 months, and he hopes to attend the wedding and dance with her. He asks you about the possibility of palliative care consultation and asks for your opinion regarding his overall prognosis. His wife and daughter are concerned that the patient is giving up and that if he discusses prognosis he will lose hope and not fight on, and thus not make it to his granddaughter's wedding.

\section{Question}

\section{Which one of the following would be the} most appropriate response to this patient?

a. "Discussing prognosis, particularly if poor, may cause depression, and you may prematurely give up."

b. "It sounds like you might be considering hospice, and I support you. May I help to make a referral for you?"

c. "If you proceed with palliative care, it is unlikely that you will be treated as aggressively as possible, and this may shorten your survival."

d. "Recent studies have shown that palliative care and chemotherapy can be used concurrently. What is your understanding of what palliative care is?"

e. "These are topics that you really should discuss with your oncologist. As your primary care doctor, I am not best able to help you in this regard."

\section{Discussion}

The patient presents with lung cancer and adverse effects that are likely attributable to both the disease and its treatment. The role of palliative care is to assist with management of symptoms and qualityof-life issues in patients with serious illness. Although some physicians and patients may worry that initiating palliative care earlier in a disease and treatment course might lead to shortened survival, a recent randomized clinical trial did not support this concern. ${ }^{6}$ Patients with advanced lung cancer who had palliative care concurrently with standard tumor-directed oncologic care had improved quality of life and symptom management, and data do not support that initiation of palliative care will shorten survival. ${ }^{6}$ It has been further demonstrated that having open and honest discussions with a patient engaged to the degree that he or she desires is not associated with loss of hope but may be life affirming and allow for decisions to be made according to the patient's understanding of the prognosis. ${ }^{7}$ It is important to recognize that hospice and palliative care are separate entities and that physicians in all specialties may have a role in maximizing a patient's quality of life in the face of serious illness. Primary care physicians retain an especially vital role in being present with patients and maintaining their therapeutic relationship in order to avoid feelings of abandonment by the patient or family.

\section{Clinical Pearl}

The involvement of palliative care has been shown to improve quality of life without worsening survival or causing patients to give up in the setting of a life-limiting illness.

\section{CASE 4}

A 79-year-old woman with moderate Alzheimer dementia is admitted to your hospital with abdominal pain. She has a history of obesity and essential hypertension controlled with oral metoprolol; she has mild chronic renal insufficiency (baseline creatinine level, $1.7 \mathrm{mg} / \mathrm{dL}$ ) and normal hepatic synthetic function. Cholecystitis is diagnosed, and the patient undergoes laparoscopic cholecystectomy. You are consulted by the surgical team to assist with medical management. The patient has been receiving oxycodone, 2.5 mg every 6 hours, and acetaminophen, $650 \mathrm{mg}$ every 4 hours, while awake for her pain. Her other medications, including metoprolol and donepezil, are continued, and "administer as needed" orders are also placed: senna for constipation, albuterol nebulization solution for wheezing, zolpidem for sleep, and sliding-scale insulin to maintain a glucose level of $150 \mathrm{mg} / \mathrm{dL}$ or less (to convert to $\mathrm{mmol} / \mathrm{L}$, multiply by 0.0555 ). The pain appears to be well controlled, but on postoperative day 3, you are called at 1 Am to evaluate her altered mental status. She has received each of her administer-asneeded medications once in the preceding 24 hours, and she is now actively hallucinating. Her vital signs include a blood pressure of 135/82 $\mathrm{mm} \mathrm{Hg}$ and a respiratory rate 16 breaths/min at rest with pulse oximetry at $94 \%$ while the patient breathes room air. 


\section{Question}

While evaluating causes of her delirium, which one of the following is the most appropriate next step in the management of her pain?

a. Discontinue oxycodone and replace it with ibuprofen, 400 mg every 6 hours for the next 48 hours

b. Discontinue oxycodone and replace it with tramadol, $50 \mathrm{mg}$ orally every 6 hours

c. Discontinue oxycodone and replace it with morphine, $5 \mathrm{mg}$ orally every 6 hours

d. Discontinue oxycodone and replace it with lorazepam, I mg intravenously once to help calm the patient. Reassess pain regimen with surgical service in the morning

e. Continue oxycodone

\section{Discussion}

This medically complex patient presents with postoperative delirium 48 hours after a cholecystectomy. She has done well on her opioid regimen to date and has excellent pain control. Although opioids are often implicated as the most likely reason for postoperative delirium, in many cases they are not the cause. ${ }^{8}$ Furthermore, uncontrolled pain may itself contribute to delirium. Although switching to morphine is a reasonable option to see if it is better tolerated by the patient, morphine is not recommended given her history of chronic renal insufficiency. Furthermore, changing to another opioid is unlikely to have a dramatic effect if other offending agents are not considered. ${ }^{1}$ Alternative causes such as coadministration of sedative-hypnotic agents or agents with anticholinergic activity need to be considered because they may precipitate delirium in patients who are receiving opioids. Nonpharmacologic causes of delirium such as urinary retention, constipation, infection, or infarction should be considered in the differential diagnosis. Use of nonsteroidal anti-inflammatory agents such as ibuprofen is not recommended given the patient's renal insufficiency. Tramadol, although often thought to be safer than traditional opiates, has more drug-drug interactions and a more unpredictable and troublesome adverse effect profile due to both its opiate and nonopiate effects, including serotonin and norepinephrine augmentation. Lorazepam may worsen delirium and cause worsening sedation or a paradoxical response of increased agitation.

\section{Clinical Pearl}

Opioids may be a cause of altered mental status in elderly patients with pain, but other causes of delirium should be considered, particularly in patients who appear to have tolerated opioids well previously.

\section{CASE 5}

An 80-year-old man with end-stage renal failure who has been undergoing hemodialysis 3 times weekly presents to your hospital with increased cough, lethargy, and fever. In the emergency department, laboratory studies demonstrate leukocytosis (white blood cell count, $23.3 \times 10^{9} / \mathrm{L}$ with a leftshifted leukocyte differential) and mixed acidosis on arterial blood gas determination $\left(\mathrm{pH}, 7.23 ; \mathrm{PO}_{2}, 52\right.$ $\mathrm{mm} \mathrm{Hg}$ [to convert to $\mathrm{kPa}$, multiply by 0.133 ] $\mathrm{PCO}_{2}$, $52 \mathrm{~mm} \mathrm{Hg}$ [to convert to $\mathrm{kPa}$, multiply by 0.133 ]; and bicarbonate, $20 \mathrm{mEq} / \mathrm{L}$ [to convert to $\mathrm{mmol} / \mathrm{L}$, multiply by 1.0]). Chest radiography reveals a large right lower lobe infiltrate suggestive of pneumonia. Health care-associated pneumonia is diagnosed, and the patient is admitted to the medical intensive care unit, where levofloxacin, meropenem, and vancomycin are administered along with noninvasive bilevel positive pressure ventilation (Bi-Pap). His condition does not improve after 48 hours, and he continues to be dyspneic, febrile, and agitated. The patient retains decision-making capacity and requests discontinuation of Bi-Pap and hemodialysis and to be kept comfortable and allowed to die. His son, who has legal power of attorney for health care, agrees that this request is consistent with his father's previously expressed wishes. The patient's wife of 62 years pleads with the son and the physician to continue intensive care measures. An empathetic discussion ensues, and later that day, the patient is taken off Bi-Pap and comfort measures are instituted. The patient receives a fentanyl infusion at 25 $\mu \mathrm{g} / \mathrm{h}$ and $25-\mu \mathrm{g}$ boluses of fentanyl intravenously every 20 minutes for pain or air hunger and subsequently dies a few hours later. The grief-stricken wife is crying at the bedside and tells her son and the attending physician, "You killed my husband!"

\section{Question \\ Which one of the following terms best describes the cause of death for this patient? \\ a. Physician aid-in-dying (also known as physician-assisted suicide) \\ b. Euthanasia \\ c. Manslaughter by the physician \\ d. Manslaughter by the physician and the patient's son \\ e. Death of natural causes (pneumonia, end- stage renal failure, respiratory failure)}

\section{Discussion}

This case illustrates the nuances of distinctions between "killing" and "allowing to die" and also the aspects of appropriate surrogate decision making. The withholding or withdrawal of life-sustaining treatments is generally accepted as permissible as an 
aspect of patient autonomy and the right to be left alone. If patients do not have decision-making capacity, their autonomy can be exercised via a surrogate decision maker. In this case, the son is the legal surrogate decision maker and appears to be acting in accord with the patient's wishes. Opioids or other medications that are used in appropriate doses to palliate symptoms, even at the risk of decreasing respiratory drive, are often cited as illustrative of the principle of "double effect." The double-effect principle contends that if a benefit (symptom relief) of a treatment outweighs an adverse effect (respiratory depression) and if intention is clear and the response is proportionate, that a treatment may be deemed morally acceptable. ${ }^{9}$ However, some investigators have recently criticized this concept as moot because studies have demonstrated that symptom palliation with opioids at the end of life does not shorten life if used appropriately (proportionately). ${ }^{10}$ The doses of the medications administered in this case seem appropriate for an opioid-naive patient and hence do not appear to represent physician aid-in-dying or homicide. The response of the patient's wife is an understandable grief response and does not necessarily place culpability for a wrongful act by the physician.

\section{Clinical Pearl}

Patients with decision-making capacity may personally request (or request via an appropriate surrogate decision maker) to withhold or withdraw life-sustaining treatments that are not consistent with their goals of care. This decision and ensuring that the patient is comfortable with appropriate analgesia constitute death from an underlying pathology (natural causes) and are not considered physician aid-in-dying.

\section{CASE 6}

A 46-year-old woman with stage III ovarian cancer presents to your outpatient clinic with nausea. She notes that the nausea has worsened over the past 2 days and that she is able to consume little more than her medications and a few sips of water without vomiting. She is receiving chemotherapy with carboplatin and paclitaxel and notes that she has not had difficulty with nausea during or after chemotherapy because her oncologist administers antiemetics prophylactically before each session. It has been 17 days since her last chemotherapy. The nausea does not seem to occur with movement, is worse after eating solids or liquids, and is accompanied by abdominal distention. She has minimal abdominal pain, which is managed with oxycodone, $10 \mathrm{mg}$ orally taken regularly 3 times a day. Her last bowel movement was 4 days ago. Abdominal radiography (obstruction series) shows no air-fluid levels, no free air, and no evidence of obstruction, but there is a moderate amount of stool in the colon.

\section{Question \\ Which one of the following is the most appropriate antiemetic to prescribe initially for this patient? \\ a. Metoclopramide, $10 \mathrm{mg}$ orally 4 times a day \\ b. Ondansetron, $8 \mathrm{mg}$ orally twice a day \\ c. Promethazine, $25 \mathrm{mg}$ orally (or by suppository) every 6 hours \\ d. Lorazepam, I mg orally every 6 hours \\ e. Granisetron, I mg orally once daily}

\section{Discussion}

The mechanism of this patient's nausea is likely gastrointestinal distention and irritation from both constipation and her ovarian cancer, and possibly a direct effect of her opioid. ${ }^{11}$ Opioid-induced nausea is mostly dopamine mediated. In this case, serotonin receptors in the gastrointestinal tract, serosa, and viscera are also involved. In addition to intensifying the patient's bowel regimen, dopamine blockade is most likely to be helpful for this patient. Metoclopramide is a dopamine $\mathrm{D}_{2}$ receptor antagonist that has some peripheral serotonin antagonism and would be the best choice. Prochlorperazine and haloperidol would also be reasonable choices.

Enough time has passed since the patient's most recent chemotherapy that it should not be a major contributor to her nausea at this time. She does not appear to have a bowel obstruction, which is important to rule out in this scenario because of the potential need for surgical intervention or nasogastric tube placement and also should be ruled out before administering metoclopramide given its promotility effects. Anxiety does not seem to be a major component of the patient's condition, and lorazepam alone has poor antiemetic effects.

Toxin-mediated nausea such as medication effects and electrolyte disturbances (eg, hypercalcemia) are mediated through the chemoreceptor trigger zone (CTZ), located at the area postrema in the floor of the fourth ventricle. The 2 neurotransmitters that are most active in the CTZ are serotonin and dopamine. Serotonin antagonists, such as ondansetron or granisetron, or dopamine antagonists, such as haloperidol, metoclopramide, or prochlorperazine, are most effective in the setting of CTZ-mediated nausea. Promethazine is a weak antagonist of dopamine, and its mechanism of action is generally through inducing sedation via antihistaminic and anticholinergic pathways. The use of promethazine is not routinely recommended for nausea given its 
limited efficacy and wide range of potential adverse effects.

\section{Clinical Pearl}

Selection of an antiemetic is best made by considering the mechanism of the drug's action and the putative factors that are contributing to the nausea.

Correspondence: Address to Keith M. Swetz, MD, MA, Division of General Internal Medicine, Section of Palliative Medicine, Mayo Clinic, 200 First St SW, Rochester, MN 55905 (swetz.keith@mayo.edu).

\section{REFERENCES}

1. Pergolizzi J, Böger RH, Budd K, et al. Opioids and the management of chronic severe pain in the elderly: consensus statement of an International Expert Panel with focus on the six clinically most often used World Health Organization Step III opioids (buprenorphine, fentanyl, hydromorphone, methadone, morphine, oxycodone). Pain Pract. 2008;8(4):287-313.

2. Ben-Aharon I, Gafter-Gvili A, Paul M, Leibovici L, Stemmer SM. Interventions for alleviating cancer-related dyspnea: a systematic review. J Clin Oncol. 2008;26(I4):2396-2404.

3. Bruera E, Sala R, Spruyt O, Palmer JL, Zhang T, Willey J. Nebulized versus subcutaneous morphine for patients with cancer dyspnea: a preliminary study. J Pain Symptom Manage. 2005;29(6):613-618.

4. Coyne PJ, Viswanathan R, Smith TJ. Nebulized fentanyl citrate improves patients' perception of breathing, respiratory rate, and oxygen saturation in dyspnea. J Pain Symptom Manage. 2002;23(2): 157-160.

5. Kamal AH, Maguire JM, Wheeler JL, Currow DC, Abernethy AP. Dyspnea review for the palliative care professional: treatment goals and therapeutic options. J Palliat Med. 2012;15(I): 106-114.

6. Temel JS, Greer JA, Muzikansky A, et al. Early palliative care for patients with metastatic non-small-cell lung cancer. $N$ Engl J Med. 2010;363(8):733-742.

7. Smith TJ, Dow LA, Virago E, Khatcheressian J, Lyckholm LJ, Matsuyama R. Giving honest information to patients with advanced cancer maintains hope. Oncology (Williston Park). 20। 0;24(6):52I-525.

8. Ganai S, Lee KF, Merrill A, et al. Adverse outcomes of geriatric patients undergoing abdominal surgery who are at high risk for delirium. Arch Surg. 2007; 142(1 I): I072-1078.

9. Sulmasy DP, Pellegrino ED. The rule of double effect: clearing up the double talk. Arch Intem Med. 1999;159(6):545-550.

10. Maltoni M, Pittureri C, Scarpi E, et al. Palliative sedation therapy does not hasten death: results from a prospective multicenter study. Ann Oncol. 2009;20(7):1 I63-1 169.

11. Wood G], Shega JW, Lynch B, Von Roenn JH. Management of intractable nausea and vomiting in patients at the end of life: "I was feeling nauseous all of the time ... nothing was working." JAMA. 2007;298(I0): I 196-1207.

\section{CORRECT ANSWERS: Case 1: b. Case 2: b. Case 3: d. Case 4: e. Case 5: e. Case 6: a}

